\title{
Aproximación multifocal para la recuperación hidromorfológica de la cuenca del río Gaià
}

\author{
GARCiA Burgos, E. ${ }^{1 *}$, BARDINA, M. ${ }^{1}$, MunNÉ, A. ${ }^{1}$, SOlÀ, C. ${ }^{1}$ \\ ${ }^{1}$ Agència Catalana de l'Aigua. Departament de Control i qualitat de les aigües. C/ Provença 204-208, 08036 Barcelona, España. \\ *evelyn.garcia@gencat.catn
}

\section{INFORMACIÓN DEL ARTÍCULO ${ }^{1}$}

Recibido el 18 de 11 de 2019

Revisión recibida el 5 de 10 de 2020

Aceptado el 1 de 12 de 2020

DOI: $10.51443 /$ Restaurarios.2020.03

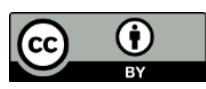

Palabras clave

Río Gaià

Custodia fluvial

Caudal ecológico

Restauración fluvial

Gobernanza.
Resumen

La cuenca del río Gaià, provincia de Tarragona, está regulada desde el año 1975 por la presencia de un embalse para uso industrial y regadío, que durante muchos años provocaba que el tramo final del río estuviera seco, y por lo tanto no llegara al mar. El régimen hídrico de la cuenca es temporal en condiciones naturales, con un régimen con un marcado carácter mediterráneo y torrencial. Desde 2011 se estableció un régimen de caudales ambientales, lo que ha permitió la recuperación de la dinámica natural en el tramo bajo del Gaià, respetando la temporalidad natural de la cuenca. Esta medida, junto con actuaciones de restauración morfológica en tramos clave como la Alameda de Santes Creus y otras actuaciones que pretenden eliminar presiones e impactos significativos, están facilitando la progresiva recuperación de la diversidad estructural y biológica del ecosistema fluvial. La custodia fluvial, como herramienta de conservación en la cuenca permite la consolidación de las actividades educativas que llevan a cabo asociaciones medioambientales como la Sínia, básicas para la gobernanza y la participación social, así como también otras actividades para evaluar la diversidad biológica y la evolución del medio fluvial en la cuenca del Gaià como el proyecto EMPESCA'T u otros estudios de seguimiento, claves para una gestión adaptativa.

La participación social ha sido muy importante para poder conseguir un consenso sobre el equilibrio entre los usos del agua asociados al embalse y el respeto de un régimen de caudales ecológico, lo que se ha conseguido gracias a las reuniones regulares de una comisión con todos los agentes sociales implicados (asociaciones ambientales, industria, regantes, ayuntamientos y administración hidráulica).

\section{Abstract}

The Gaià river basin, located in the province of Tarragona, has been regulated since 1975 by the presence of a reservoir for industrial and irrigation use, which for many years dried the final stretch of the river, which therefore did not reach the sea. The hydrological regime is temporary in natural conditions, with a marked Mediterranean and torrential character. An environmental flow regime, established since 2011, has allowed the recovery of the natural dynamics in the lower section of the Gaià, respecting the natural temporality of the basin. Since 2011, there is an agreement of releasing environmental flows that has allowed the river to recover certain ecological processes. This measure, along with other morphological restoration projects on key reaches as the Santes Creus, as well as with other planned actions related to longitudinal and transversal connectivity will allow the river ecosystem to recover the structural and biological diversity. Finally, river stewardship, as conservation tool, allows environmental NGO's to reinforce educational activities which are basic for public participation and governance and are also important to establish programs (as the EMPESCA'T project or others) to monitor biological diversity and ecological status of the Gaia river. These programs are important for an adaptive management of the river.

Social participation has been very important in order to achieve a consensus on the balance between water uses and the respect of an environmental flow regime, which has been achieved thanks to the regular meetings of a commission with all the social agents involved (environmental associations, industry, irrigators, municipalities and water authority). 


\section{Introducción}

Los ríos temporales son ecosistemas fluviales vulnerables, hábitat de especies de fauna y flora de distribución reducida o algunas incluso endémicas de cada cuenca.

Sin embargo, estos cursos han sido frecuentemente olvidados, tanto en el campo de la ciencia como de la gestión, al ser considerados sistemas de poco valor ecológico y económico (Larned et al, 2010). Por ejemplo, en los procesos de intercalibración para la evaluación de estado ecológico estos ríos no se han tenido en cuenta, ya sea porque suelen presentar una cuenca de drenaje poco importante como por la dificultad de muestrear sus cauces en un momento en el que se encuentre flujo de agua.

Por estas mismas razones, la aplicación de medidas de restauración en ríos temporales no ha sido una prioridad para los organismos competentes, dado que no existen suficientes datos para su caracterización y diagnosis. Sin embargo, en los últimos años, se han producido ciertos avances encaminados a establecer metodologías de evaluación del estado ecológico que se adapten a esta tipología de río (regímenes temporales), como son los estudios que se derivan del proyecto LIFE TRIVERS (http://www.lifetrivers.eu/es/).

En esta comunicación se detalla la aplicación de medidas para la recuperación hidromorfológica del río Gaià, un río temporal, en que han confluido múltiples actores territoriales donde han confluido las sinergias de múltiples actores territoriales. El seguimiento muestra una tendencia positiva en los elementos de valoración del estado ecológico, pero también de la complejidad de la estructura y de la diversidad biológica, aportando información de gran utilidad para una gestión adaptativa de la cuenca.

\section{Contexto físico y social en la cuenca del Gaià}

\section{$2.1 \quad$ Antecedentes}

La cuenca del río Gaià, con un área de drenaje de $433 \mathrm{~km}^{2}$, está situada en la provincia de Tarragona (Figura 1). Atraviesa la sierra Litoral por un estrecho en el que se localiza el pantano del Catllar, construido en el año 1975, para procurar a REPSOL una reserva estratégica de agua para la industria petroquímica de Tarragona, así como pequeños regadíos locales de la zona del bajo Gaià, que ocupan en el municipio de la Riera de Gaià una superficie agrícola de 406 ha. A raíz de la construcción del embalse, los últimos $11 \mathrm{~km}$ de río normalmente discurrían secos durante gran parte del año. El embalse tiene una capacidad de $57 \mathrm{hm}^{3}$, aunque desde 2010 el volumen máximo que ha alcanzado es de $9,72 \mathrm{hm}^{3}$, lo que representa un $17 \%$ de su capacidad.

El río desemboca en el municipio de Altafulla, formando una laguna temporal separada del mar por una barra de arena que se forma por la dinámica litoral natural. Esta laguna y sus alrededores forman parte de la Reserva Natural de Fauna Salvaje de la Desembocadura del Gaià.

El régimen hidrológico natural de la cuenca sin tener en cuenta la presión del pantano sería de régimen temporal, con un comportamiento estacional en verano en que quedaría seco o con lagunas intermitentes en el curso medio y bajo. La aportación de agua superficial que entra por la cola del embalse es de media de $9,8 \mathrm{hm}^{3}$ anuales (datos 1996-2008), pero como otras cuencas de régimen mediterráneo presenta crecidas torrenciales, que regionalmente se conocen como Gaianadas. Por lo que la aportación media anual puede oscilar desde $1,34 \mathrm{hm}^{3}$ en el año hidrológico 2016-17 y los 22,49 hm ${ }^{3}$ en el año 201920.

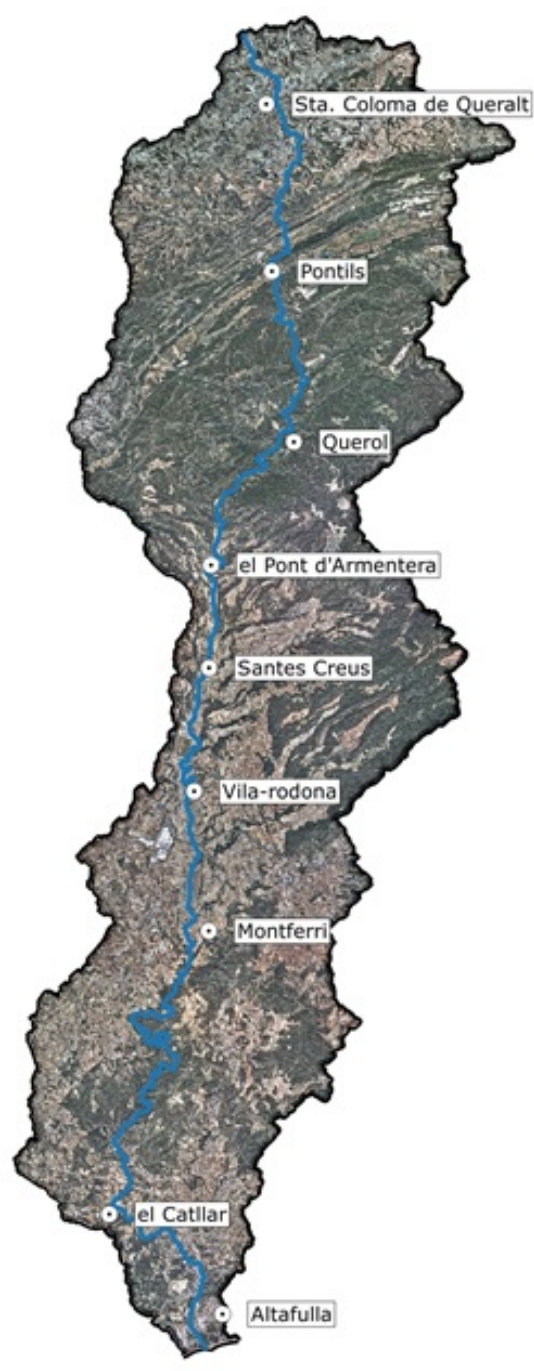

Figura 1 Cuenca del Gaià. 
Tabla 1 Calidad de las masas de agua del eje fluvial del Gaià, evaluada con datos de 2007 a 2012 (Fuente: Agència Catalana de l'Aigua, 2015).

\begin{tabular}{|c|c|c|c|c|c|}
\hline Nombre de la masa (y código) & Tipología & $\begin{array}{l}\text { Calidad } \\
\text { Biológica }\end{array}$ & Calidad FQ & $\begin{array}{l}\text { Calidad } \\
\text { HIMO }\end{array}$ & $\begin{array}{c}\text { Estado } \\
\text { ecológico }\end{array}$ \\
\hline $\begin{array}{l}\text { Cabecera del Gaià hasta Pontils, } \\
\text { incluido el río Boix (0600010) }\end{array}$ & $\begin{array}{l}\text { Ríos mediterráneos de } \\
\text { caudal variable (R-T09) }\end{array}$ & Buena & $\begin{array}{l}\text { Inferior a } \\
\text { buena }\end{array}$ & $\begin{array}{l}\text { Inferior a } \\
\text { buena }\end{array}$ & Mediocre \\
\hline $\begin{array}{l}\text { El Gaià de de Pontils a la cola del } \\
\text { embalse del Catllar }(0600020)\end{array}$ & $\begin{array}{l}\text { Ríos de zonas cársticas } \\
\text { (R-T10) }\end{array}$ & Buena & Buena & Buena & Bueno \\
\hline $\begin{array}{l}\text { El Gaià des de la presa del Catllar } \\
\text { hasta el mar }(0600070)\end{array}$ & $\begin{array}{l}\text { Ríos de zonas cársticas } \\
\text { (R-T10) }\end{array}$ & Sin controles & Sin controles & Sin controles & Sin controles \\
\hline
\end{tabular}

La explotación típica del embalse hasta el año 2010 era de aproximadamente de $3,6 \mathrm{hm}^{3}$ para uso industrial de REPSOL (37\%), y de 0,6 $\mathrm{hm}^{3}$ para los regantes (6 \%). En este embalse la infiltración es significativa: la infiltración y la evaporación se estiman, en conjunto, en unos 5,6 hm $\mathrm{hm}^{3}$ de media (57\%).

En el año 2010 la Agència Catalana de l'Aigua (Agència en adelante) empezó un proceso para elaborar una hoja de ruta que permitiera un manejo más sostenible de los caudales de la presa del Catllar que finalizó con la firma de un convenio entre la Agència y REPSOL por el que se libera un régimen de caudales ambientales mínimos, así como caudales generadores, tal como se expondrá más adelante.

Según la planificación hidrológica, el eje principal de la cuenca es el río Gaià se divide en 3 masas de agua, tal y como se detalla en la Tabla 1 , donde se asocia con la calidad de los diferentes elementos del estado ecológico.

La cuenca del Gaià cuenta con 5 espacios dentro de la Red Natura 2000, entre los que destaca la alameda de Santes Creus y la laguna de la desembocadura.

Desde el 2006 se han llevado a cabo diversas actuaciones para la recuperación del río (Tabla 2), tanto por parte de la Asociación la Sínia, pionera en actuaciones de custodia fluvial como por parte de la Agència.

\subsection{Mapa de actores}

En el desarrollo de las diferentes actuaciones para la recuperación del río Gaià se pueden identificar los siguientes actores principales:

La Asociación mediambiental la Sínia, que nace en el 1997 para promover la educación ambiental en la cuenca del río Gaià, actualmente desarrollando el proyecto "Amigos $\mathrm{y}$ amigas del Gaià, red de voluntariado ambiental del curso bajo del río Gaià" y responsable del acuerdo de custodia fluvial del dominio público en toda la cuenca. Forma parte también de la plataforma Salvem el Gaià, y ambos son elementos clave para la custodia del territorio.

La Coordinadora de Salvem el Gaià nació el 1994 para dar visibilidad a la problemática de falta de caudal del río, y que fue apoyada por 50 entidades del Baix Gaià y todos los ayuntamientos de la comarca que dieron apoyo a la recuperación del río, con campañas de recogida de firmas. Toda esta campaña culminó con la firma del convenio entre REPSOL y la Agència para la implantación del régimen de caudales ecológicos. Desde el 2011 la coordinadora pasa a ser l'Associació per les Terres del Gaià, cuyo rol es más divulgativo, ya que organiza, por ejemplo, una marcha popular "la marxa per les Terres del Gaià" que moviliza centenares de personas.

Tabla 2 Principales actuaciones desarrolladas en la cuenca del Gaià.

\begin{tabular}{lcc}
\hline $\begin{array}{l}\text { Actuación } \\
\text { Restauración de las terrazas aluviales de l'albereda de } \\
\text { Santes Creus }\end{array}$ & Localización & \multicolumn{1}{c}{ Procedimiento } \\
$\begin{array}{l}\text { Implantación de caudales ecológicos } \\
\text { Recuperación de núcleos de ribera autóctonos y } \\
\text { control de caña (Arundo donax) }\end{array}$ & Toda la cuenca & Subvención \\
$\begin{array}{l}\text { Dragado del humedal del Catllar y mejora del by-pass } \\
\text { de sedimento }\end{array}$ & Tramo bajo y el Catllar & Convenio REL de custodia fluvial \\
$\begin{array}{l}\text { Mantenimiento de senderos } \\
\text { Recuperación de hábitats para la fauna acuática y }\end{array}$ & Tramo bajo & Convenio de custodia fluvial \\
control de especies invasoras de fauna & Toda la cuenca & Convenio de custodia fluvial \\
\hline
\end{tabular}


Esta asociación promueve la conservación y la divulgación de los valores ambientales e históricos de la zona.

REPSOL es la empresa propietaria de la presa del Catllar, que se utiliza como reserva de agua estratégica para la refrigeración de la planta de producción situada en el campo de Tarragona. Como fuente de abastecimiento alternativa cuentan con la que abastece el Consorcio de Aguas de Tarragona, proveniente de la cuenca del Ebro, o bien aguas regeneradas de la EDAR de Altafulla.

Las comunidades de regantes del Catllar, la Riera de Gaià y Altafulla-Tamarit comparten con REPSOL el uso del agua del embalse del Catllar, siendo el uso de riego prioritario frente al uso industrial. La concesión otorga un derecho de hasta $11 \mathrm{hm}^{3}$ a uso industrial y de $3 \mathrm{hm}^{3}$ a regadío. La captación se realiza en la presa, y se distribuye mediante tuberías.

La Diputació de Tarragona promueve la recuperación y el uso público del espacio fluvial en la cuenca mediante proyectos emblemáticos como el EMPESCA'T que lleva a cabo la asociación la Sínia y otros más turísticos como el camino azul. También es propietaria del área recreativa de la Alameda del Monasterio de Santes Creus.

La Agència es el organismo competente en la gestión del agua y los ecosistemas acuáticos en la cuenca del Gaià, y por lo tanto establece los objetivos ambientales en el plan de gestión, y programa y financia las medidas necesarias para conseguirlos. En el segundo ciclo de planificación (2016-2021) se programaron, entre otras, medidas de saneamiento, promoción de la custodia fluvial, así como actuaciones de recuperación de la morfología y las riberas.

Finalmente, los Ayuntamientos también son parte interesada, tanto en la gestión del embalse como en las medidas que se aplican en la cuenca.

La coordinación entre todos estos actores se realiza mediante diferentes mecanismos:

- El acuerdo entre la Agència y REPSOL, establece la creación de una comisión de seguimiento formada por representantes de ambos, que se reúne regularmente para evaluar los resultados de la gestión del embalse.

- Se ha creado una comisión de participación con el resto de actores del bajo Gaià (Ayuntamientos, regantes, y entidades ambientales), que se reúne anualmente, y en la que se informa de las entradas y salidas del embalse en cada año hidrológico, y se debaten las posibles mejoras en la gestión, así como la evolución del convenio, de modo que estos actores pueden incidir realmente, como por ejemplo modificando la consigna de cotas que condicionan la liberación de caudales ambientales. Este esquema mediante comisiones de participación para el seguimiento de la evolución del régimen de caudales ecológicos no se conoce que se haya implementado en otros casos similares.

- El diseño de los programas de medidas que realiza la Agència se somete también debate en los procesos de participación que van asociados a cada ciclo de planificación, que se ha implementado en todas las Demarcaciones hidrográficas, ya que responde a requerimientos de la Directiva Marco del Agua.

- Los proyectos de restauración promovidos por la Agència siguen el procedimiento regulado de información pública. Los proyectos de restauración promovidos por las entidades ambientales se enmarcan en el acuerdo de custodia firmado con la Agència, y pueden optar a líneas de subvenciones que esta promueve para conseguir financiación.

- La Diputació de Tarragona juntamente con todos los alcaldes de los ayuntamientos de la cuenca conforman la mesa del Gaià, en la que debaten proyectos emblemáticos a escala de cuenca.

\section{Material y métodos}

\subsection{Desarrollo de la custodia fluvial}

Desde el año 2016 la Agència dispone de un modelo de convenio de custodia fluvial consensuado con la Xarxa de Custòdia del Territori que permite la custodia del dominio público hidráulico basado el desarrollo de un Programa de acción de 4 años en el ámbito de custodia. La custodia fluvial es una herramienta de co-gestión entre la administración hidráulica y una entidad local o supralocal que se implica en el desarrollo de medidas de gestión y de actuación a cambio de mayor agilidad en la tramitación administrativa, apoyo técnico y apoyo económico. Este procedimiento es el que, desde el año 2017, se ha aplicado en un tramo de $65 \mathrm{~km}$ de longitud del dominio público hidráulico del río Gaià mediante un convenio de custodia con la Asociación medioambiental la Sínia, y cuyas actuaciones se iniciaron el año 2019. El Programa de acción incluye actuaciones para la recuperación del bosque de ribera (debido a que la caña americana ha conseguido desestructurarlo), recuperación de hábitats fluviales, seguimiento de la biodiversidad de la cuenca (ictiofauna y réptiles acuáticos) y la promoción de un uso social sostenible. El convenio de custodia permite disponer de un marco legal con reconocimiento de la administración por las actuaciones que lleva desarrollando la Sínia desde hace años. 


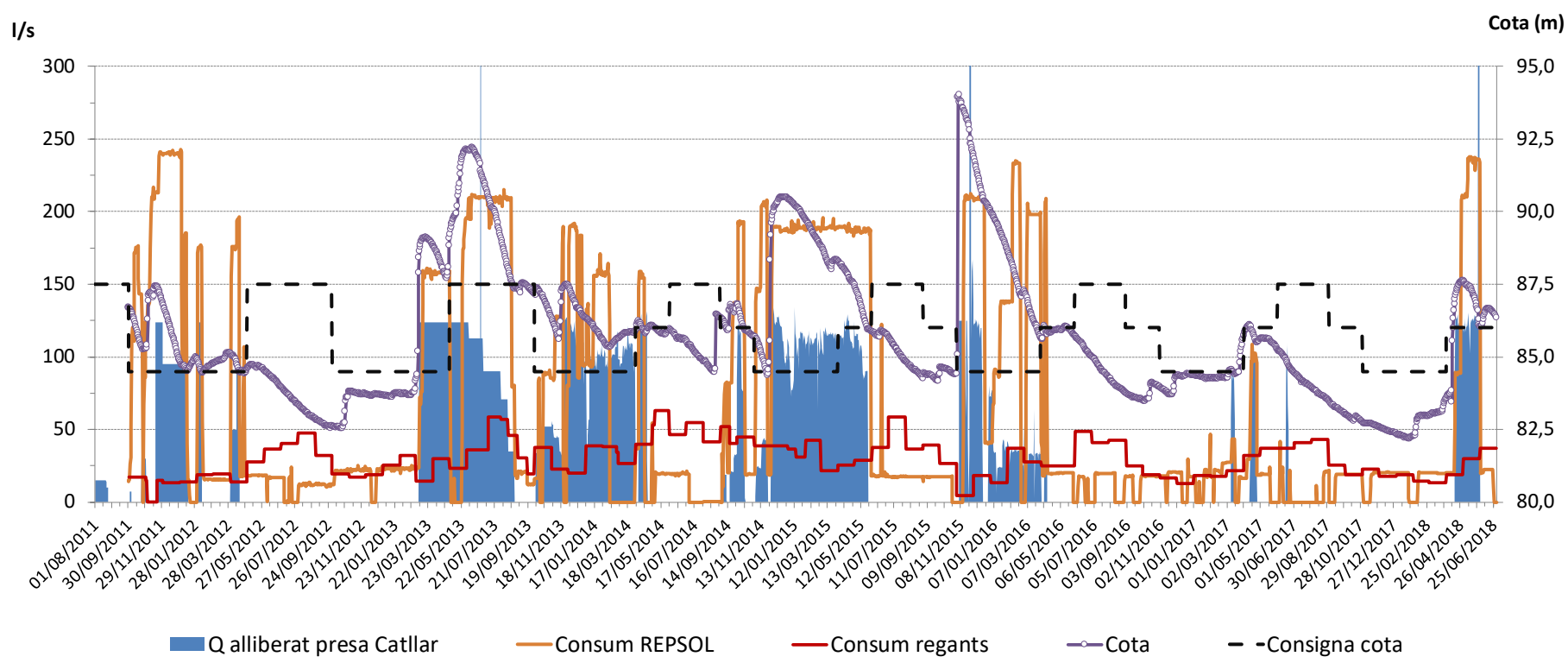

Figura 2 Cota del embalse, consumos y caudales ambientales liberados.

\subsection{Actuaciones en la cuenca}

\subsubsection{Implantación de caudales ecológicos}

Gracias a la aplicación de la Directiva Marco del Agua pero, sobre todo, a la presión del tejido social ejercida por las organizaciones Salvem el Gaià y la Associació per les Terres del Gaià, se implantaron unos caudales ecológicos desde el año 2011 en el tramo bajo del río (desde la presa del Catllar hasta la desembocadura), una medida que ha sido clave para sostener la auto-recuperación del ecosistema fluvial. Desde el año 2018 los caudales ecológicos son de obligado cumplimiento en toda la cuenca, tal como establece el Decreto 1/2017 por el que se aprobó el Plan de gestión del distrito de cuenca fluvial de Catalunya.

El convenio establece que se liberaran caudales ecológicos en función de la cota a la que se encuentre el embalse. Esta consigna de cota del embalse varía de mes en mes oscilando entre $84,5 \mathrm{msnm}$ en invierno y 87,5 msnm en verano, así como el caudal ecológico que se libera, que está entre 90 y 124 1/s. Así pues, el embalse se gestiona mediante un modelo que tiene en cuenta las entradas y salidas de la presa, los usos (industrial y de regadío), así como la evapotranspiración y la infiltración, siendo esta última significativa cuando el embalse supera la cota $85 \mathrm{msnm}$. En los periodos en los que la cota del embalse está por debajo de la consigna, el uso industrial no deriva agua, y se abastece del Consorcio de aguas de Tarragona. En estos casos, también existe una alternativa para el uso industrial consistente en consumir las aguas reutilizadas de la EDAR de Altafulla.

La explotación del embalse por parte de la industria ha cambiado respecto la realizada con anterioridad a la firma del convenio, desde 2011 hasta 2019 su consumo ha oscilado entre $0,5 \mathrm{hm}^{3}$ el año más seco hasta $3,54 \mathrm{hm}^{3}$ en el año de mayor disponibilidad en el embalse. Por parte de los regantes, el consumo es más estable, variando entre 0,7 y 1,2 hm³/año. El régimen de caudales ambientales también ha sido variable, con un mínimo de $0,19 \mathrm{hm}^{3} / \mathrm{año}$ y un máximo de $2,77 \mathrm{hm}^{3} /$ año.

En la Figura 2 se representan los niveles de consumo y la variación de la cota de la presa, así como las consignas correspondientes en el período 2011-2018.

La liberación de caudales ambientales varía según las aportaciones que recibe la cuenca, de modo que en años húmedos se han implementado los caudales ecológicos mínimos hasta un $60 \%$ del tiempo, mientras que en años secos únicamente se han podido liberar en un $7 \%$ del tiempo, ya que los bajos niveles del embalse no lo permitieron, reservándose el agua para el uso industrial y de riego. En cuanto al volumen, el caudal ambiental oscila entre el 12 y el 36\% de la aportación anual que ha recibido el embalse (Tabla 3 ).

Los caudales ambientales se completaron con caudales generadores; estos últimos enfocados a la movilización del lecho fluvial (transporte de sedimento) y la regeneración hidromorfológica del cauce aguas abajo de la presa. En los 10 años de vigencia del convenio se han liberado caudales generadores en 5 ocasiones (cuando las reservas de agua del embalse lo permitían), con caudales máximos entre 2 y $3 \mathrm{~m}^{3} / \mathrm{s}$ mantenidos durante 24 a 48 horas. Estos caudales permitieron en 2016, 2018 y 2020 que el río Gaià llegara hasta la desembocadura, lo que no ocurría desde la puesta en marcha del embalse. Los caudales generadores, conseguidos abriendo compuertas de fondo después de episodios de grandes avenidas en la cuenca, lograron aportar materiales finos al tramo final 
Tabla 3 Evolución anual de las aportaciones y del caudal ambiental.

\begin{tabular}{|c|c|c|c|c|c|}
\hline Año hidrológico & $\begin{array}{l}\text { Aportación EA } \\
\text { Vilabella }\left(\mathrm{hm}^{3}\right)\end{array}$ & $\begin{array}{l}\text { № días } Q \\
\text { ambiental }\end{array}$ & $\begin{array}{c}\text { Q ambiental } \\
\text { liberado }\left(\mathrm{hm}^{3}\right)\end{array}$ & $\begin{array}{l}\text { Liberación caudal } \\
\text { (\% tiempo) }\end{array}$ & $\begin{array}{l}\text { Liberación caudal } \\
\text { (\% aportación) }\end{array}$ \\
\hline 2011-12 & 2,7 & 98 & 0,7 & $27 \%$ & $27 \%$ \\
\hline $2012-13$ & 8,1 & 172 & 1,7 & $47 \%$ & $21 \%$ \\
\hline 2013-14 & 4,3 & 220 & 1,5 & $60 \%$ & $36 \%$ \\
\hline 2014-15 & 6,7 & 213 & 1,8 & $58 \%$ & $27 \%$ \\
\hline $2015-16$ & 4,2 & 144 & 0,68 & $39 \%$ & $16 \%$ \\
\hline $2016-17$ & 1,34 & 26 & 0,19 & $7 \%$ & $14 \%$ \\
\hline 2017-18 & 9,81 & 123 & 1,42 & $34 \%$ & $14 \%$ \\
\hline 2018-19 & 8,49 & 169 & 1,74 & $46 \%$ & $20 \%$ \\
\hline 2019-20 & 22,49 & 268 & 2,77 & $73 \%$ & $12 \%$ \\
\hline
\end{tabular}

del río, muy necesarios para restablecer el equilibro sedimentario, que se había visto alterado por la regulación ejercida por el embalse. Estos caudales resultaron ser un elemento clave para transformar la dinámica fluvial aguas abajo del embalse, más importantes incluso que el régimen de caudales mínimos. El régimen de caudales mínimos liberados los primeros años se infiltraba en los primeros kilómetros, de modo que no consiguió llegar al mar hasta que los caudales generadores fueron recubriendo de materiales finos y restableciendo el equilibrio sedimentario del lecho del río.

\subsubsection{Desarrollo de actuaciones morfológicas de restauración pasiva}

Los proyectos de restauración deberían focalizarse a la eliminación de presiones e impactos, basándose en el principio de mínima intervención y recuperar el funcionamiento de estos ecosistemas a un estado más natural o equivalente al que tenían antes de su deterioro (González del Tánago et al., 2007). Siguiendo esta premisa, se han desarrollado intervenciones en la cuenca, alguna de ellas ejecutada, otras en proceso de ejecución y algunas más planificadas, que pretenden aplicar esta definición de restauración. En el primer ciclo (2009-2015) de la planificación se desarrolló la restauración morfológica en la alameda de Santes Creus.

La alameda de Santes Creus en el municipio de Aiguamúrcia es probablemente el paisaje fluvial con mayor simbolismo por sus referencias históricas y por haber sido declarado el primer espacio natural fluvial de Catalunya. En trabajos de la planificación de espacios fluviales se identificó como el tramo más degradado de la cuenca media y alta, los cuales se caracterizaban por una conservación ecológica superior a otras cuencas del Distrito de cuenca fluvial de Catalunya. Por esta razón se llevó a cabo la restauración morfológica de un tramo canalizado con escollera mediante su eliminación y reconexión de las terrazas con el lecho. La canalización había provocado la desconexión de las llanuras aluviales con el cauce, con la correspondiente incisión y empobrecimiento de los hábitats fluviales asociados. Los datos principales del proyecto se detallan en la Tabla 4.

Tabla 4 Principales datos del proyecto de restauración de la alameda de Santes Creus.

Datos principales
Inicio: octubre $2010 \quad$ Final: junio 2011
Longitud: $1,2 \mathrm{Km}$
Presupuesto proyecto: $435.504,7$ euros
Volumen escollera eliminado: $2.986 \mathrm{~m} 3$

La actuación siguió el principio de restauración pasiva, por lo que no se efectuó ninguna plantación una vez realizados los movimientos de tierra, así como tampoco se diseñó un lecho de aguas bajas.

En noviembre de 2015 se registró una Gaianada, con caudal máximo de $75 \mathrm{~m}^{3} / \mathrm{s}$ (que correspondería a un período de retorno de alrededor de100 años) y un calado máximo por encima de la lámina de agua en régimen normal de 4,5 metros. Esta crecida permitió la movilización del material del lecho: el transporte del sedimento producido es la causa de la configuración actual del río, con un cauce de aguas bajas estrecho y reconectado con la llanura aluvial. Asimismo, la vegetación ruderal y las especies invasoras que cubrían una parte del cauce fueron eliminadas, dando paso a playas de gravas y arenas (Figura 3 ).

En el segundo ciclo de planificación (2016-21) están en desarrollo otras actuaciones cuyo objetivo es la eliminación de presiones e impactos como la eliminación del colector de aguas residuales entre el núcleo del Catllar y la desembocadura (con una longitud aproximada de $7.300 \mathrm{~m}$ ) y la eliminación de las estaciones de aforo en desuso de Querol y de Vespella, que permitirán retirar estructuras que interfieren en la dinámica natural del río. 


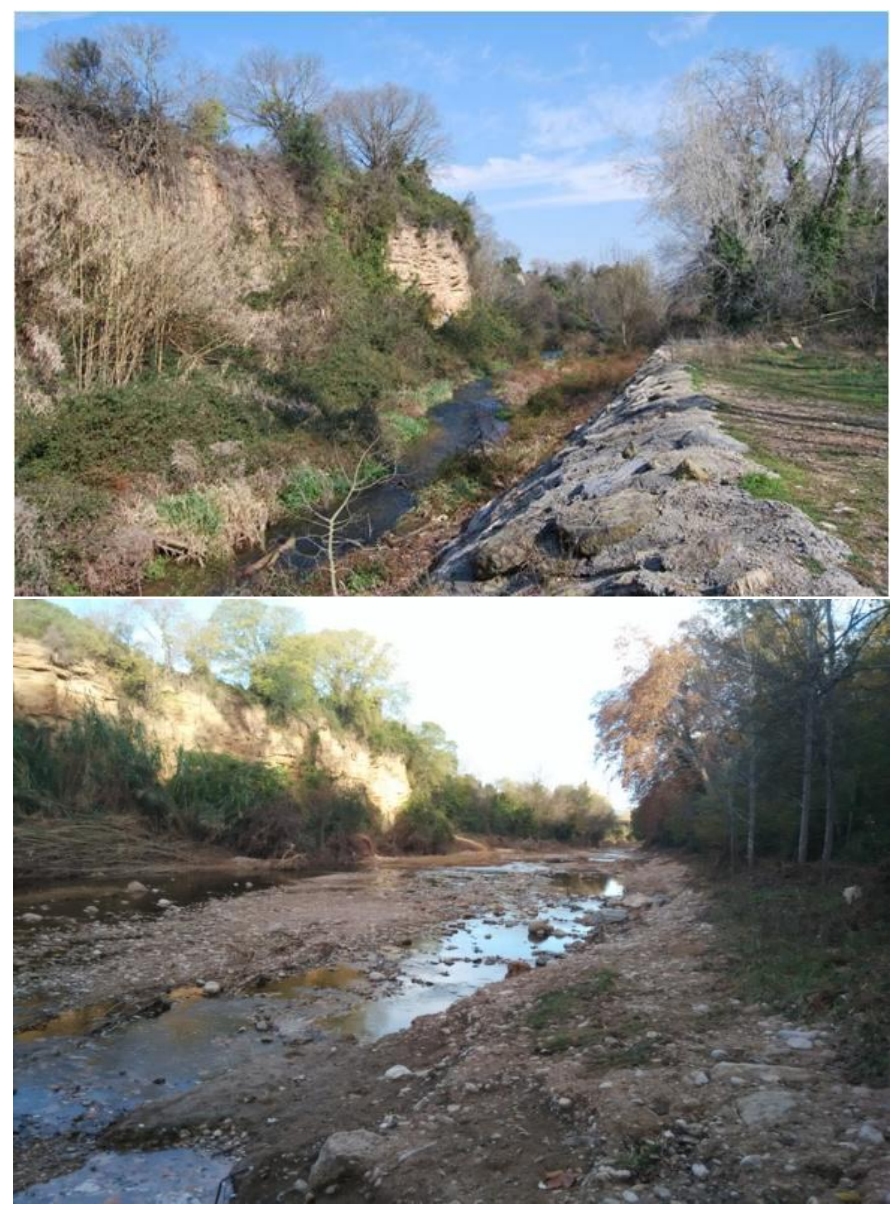

Figura 3 Imagen del margen derecho antes del proyecto (arriba) y después del proyecto de restauración de la alameda de Santes Creus, una vez pasada la Gaianada (abajo).

\section{Resultados}

\subsection{Evolución de la diversidad estructural del ecosistema fluvial en la alameda de Santes Creus}

El Programa de seguimiento de la restauración del meandro de Santes Creus (2010-2020), diseñado por la Diputació de Tarragona tiene en cuenta el principio de restauración pasiva. Los elementos que se incluyen en el programa, y que permiten apreciar los cambios producidos sobre la estructura física y la diversidad biológica son: topografía, secciones hidromorfológicas, seguimiento del paisaje fluvial, cartografía de hábitats a escala 1:2.500, riqueza florística e indicadores biológicos (macroinvertebrados y diatomeas). Estos indicadores son complementados por otros dos elementos que aportan información sobre la diversidad biológica y la complejidad estructural: la cartografía de los hábitats y la riqueza florística. De este modo, al analizar la riqueza florística (especies de flora) en el ámbito restaurado, se verifica la correspondencia clara entre la recuperación topográfica del río, desde la finalización del proyecto, con la riqueza ecológica, que ha permitido la recolonización del ámbito por un número importante de especies de flora, con 33 nuevos taxones de flora vascular citados, asociados a los arenales y herbazales ruderales húmedos, tal y cómo muestra la Tabla 5.

Tabla 5 Evolución de los taxones de flora en la alameda de Santes Creus según los diferentes estudios. *DIPTA: Diputació de Tarragona.

\begin{tabular}{lcccc}
\hline $\begin{array}{l}\text { Estudios Riqueza } \\
\text { de flora }\end{array}$ & $\begin{array}{c}\text { Salat } \\
\mathbf{( 1 9 9 1 )}\end{array}$ & $\begin{array}{c}\text { DIPTA* } \\
\text { (2009) }\end{array}$ & $\begin{array}{c}\text { DIPTA } \\
\mathbf{( 2 0 1 2 )}\end{array}$ & $\begin{array}{c}\text { DIPTA } \\
\text { (2016) }\end{array}$ \\
$\begin{array}{l}\text { Taxones en el } \\
\text { ámbito del proyecto }\end{array}$ & 84 & 122 & 149 & 154 \\
\hline
\end{tabular}

Esta evolución de la complejidad florística también se puede observar en la cartografía de los hábitats fluviales, tal y cómo muestra la Figura 4.

Los hábitats fluviales se han incrementado a medio plazo, esperable tras una actuación de restauración como la de referencia, aunque la curva de crecimiento tiende a aplanarse hasta que llega una nueva perturbación que genera nuevos espacios abiertos y diversidad topográfica, cómo la Gaianada del 2015.

\subsection{Seguimiento de la biodiversidad faunística de la cuenca}

La evaluación de la distribución, composición, abundancia y dinámica poblacional de determinadas especies de fauna acuáticas clave en la cuenca es un elemento de gran interés para evaluar su estado de conservación en relación con las presiones e impactos que afectan a la cuenca y cómo evolucionan cuando se llevan a cabo actuaciones de recuperación de las dinámicas fluviales.

La asociación La Sínia en el ámbito de la custodia fluvial y con el apoyo de la Diputació de Tarragona lleva a cabo en el Baix Gaià el programa Empesca't. Este programa comporta el seguimiento de la biodiversidad del espacio fluvial de especies acuáticas clave: peces, anfibios y réptiles.

La presa del Catllar interrumpe completamente la conectividad fluvial con una población de peces que se estructura en dos bloques independientes sin asociación alguna: un bloque compuesto por el tramo de cabecera y el tramo medio, y el otro bloque formado por el bajo Gaià, por lo que no se puede hablar de una comunidad íctica sino de poblaciones aisladas. En el tramo alto y medio el barbo colirrojo (Barbus haasi), es la especie dominante y presenta un estado de conservación cercano a bueno (mejor en la cabecera que en el tramo medio), mientras que el bagre (Squalius cephalus), con una distribución más acotada, muestra un estado de conservación moderado.

Por otro lado, la anguila (Anguilla anguilla) se presenta como especie acompañante y esporádica en el tramo medio, con un estado de conservación deficiente. En este sector de la cuenca, la ausencia de núcleos reproductores de especies exóticas genera una integridad biótica de las comunidades ictícolas. 

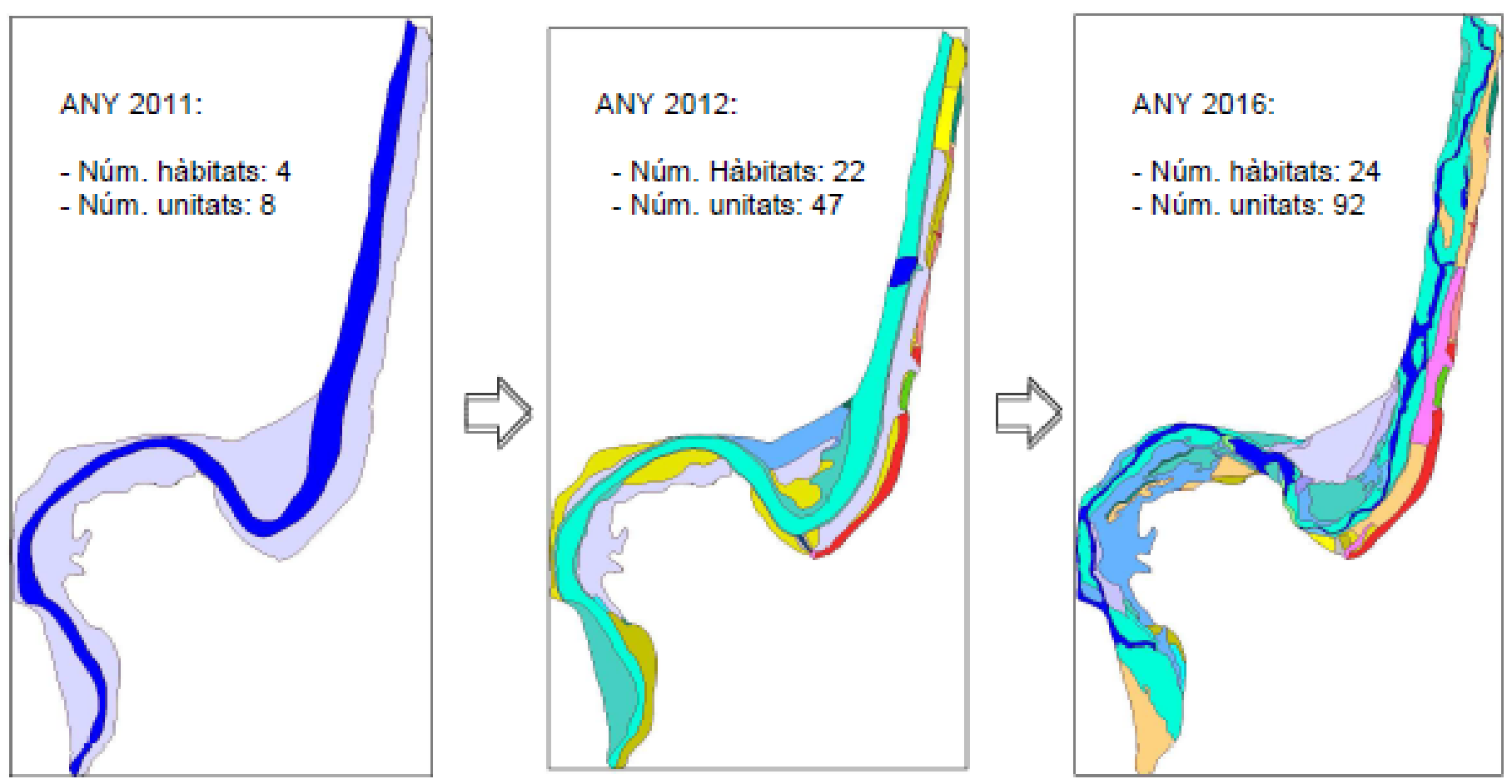

Figura 4 Evolución de los hábitats fluviales y las unidades cartografiadas en el ámbito de la alameda de Santes Creus justo acabado el proyecto y hasta el año 2016, después de la Gaianada (Fuente: Diputació de Tarragona, 2016)

En el tramo bajo del Gaià, la comunidad íctica presenta un estado de conservación deficiente, con presencia puntual de mugílidos asociada a episodios de intensa intrusión salina en la desembocadura. Sólo la gambusia (Gambusia holbrooki) presenta un núcleo reproductor en este ámbito, siendo la especie invasora más relevante, con alguna carpa (Cyprinus carpio) y alburno (Alburnus alburnus), juntamente con la carpa dorada (Carassius auratus).

Desde 2011, la implementación de los caudales ecológicos ha permitido, aunque esporádicamente, la conexión total del curso fluvial con la desembocadura. Todavía no se dispone de suficientes datos sobre la evolución de las poblaciones de peces, y el carácter temporal del régimen hidrológico limitará la recuperación de estas comunidades. Pero la reconexión con el mar ha permitido recuperar tramos de inundación permanente que pueden acoger núcleos poblacionales de algunas especies potencialmente presentes en el tramo bajo del río (anguila, fartet o barbo). En el 2018 se detectó puntualmente la presencia de anguila en una estación de control del tramo bajo del Gaià, y la hipótesis principal es que su presencia se debe a su ascenso desde el mar en un momento en el que había suficiente caudal.

\subsection{Estado ecológico de las masas de agua de la cuenca del Gaià}

El programa de seguimiento y control de las masas de agua es una herramienta básica para la gestión de la cuenca a una escala que permite la detección de impactos $\mathrm{y}$ presiones, de forma que se puedan planificar medidas para su corrección y la consecución de los objetivos de calidad que posee cada masa de agua. Asimismo, los resultados que se obtienen en los puntos de control en cada masa de agua también pueden detectar eventos extremos o la aplicación de medidas con una repercusión más allá del tramo en que se aplica, como puede ser la recuperación de caudales ecológicos o el efecto de una Gaianada.

Sin embargo, la escala geográfica de los puntos de control es una limitación para poder establecer la correspondencia entre la mejora de los diferentes elementos que conforman el estado ecológico y las actuaciones morfológicas que se desarrollan en la cuenca, por lo que normalmente sólo es posible detectar cambios asociados a algún indicador o parámetro de los elementos que conforman el estado ecológico en las masas de agua del Gaià (Tabla 6).

De los valores recopilados desde el 2015 por el Programa de seguimiento y control se puede apreciar una tendencia a la mejora de los indicadores biológicos y del indicador de la diversidad física del cauce (IHF). También, cabe destacar la disminución del índice QBR (Munné et al., 2003) que se puede relacionar en el tramo alto y medio con la Gaianada que ocurrió a finales del 2015 y que resultó en una potencia erosiva significativa. 
Tabla 6 Calidad de los elementos biológicos y hidromorfológicos en las masas de agua del eje principal del Gaià. Se indica el valor de referencia de los índices biológicos IBMWP e IPS, así como el nivel de calidad resultante: MB muy bueno, B bueno, M moderado, D deficiente (Fuente: Agència Catalana de l'Aigua, 2011).

\begin{tabular}{lcccc}
\hline Masa de agua & $\begin{array}{c}\text { Macroinvertebrados } \\
\text { (IBMWP) }\end{array}$ & $\begin{array}{c}\text { Diatomeas } \\
\text { (IPS) }\end{array}$ & Hábitat fluvial (IHF) & Bosque de ribera (QBR) \\
Valor de referencia & 133 & $17 / 19$ & No aplica (0-100) & No aplica (0-100) \\
\hline $\mathbf{0 6 0 0 0 1 0}$ & $122(\mathrm{MB})$ & $14,7(\mathrm{~B})$ & 78 & $75(\mathrm{~B})$ \\
2015 & $142(\mathrm{MB})$ & $9,6(\mathrm{M})$ & & $50^{*}(\mathrm{D})$ \\
2016 & $156(\mathrm{MB})$ & $14,4(\mathrm{~B})$ & & $70(\mathrm{M})$ \\
2017 & & $14,3(\mathrm{~B})$ & 67 & $45^{*}(\mathrm{D})$ \\
2018 & & & 69 & \\
\hline $\mathbf{0 6 0 0 0 2 0}$ & $106(\mathrm{~B})$ & $12,2(\mathrm{M})$ & & \\
2015 & $84(\mathrm{M})$ & $11,2(\mathrm{M})$ & & \\
2016 & $116(\mathrm{~B})$ & $9,9(\mathrm{M})$ & & \\
2017 & & $13,2(\mathrm{M})$ & & \\
2018 & & & & \\
\hline $\mathbf{0 6 0 0 0 7 0}$ & $76(\mathrm{M})$ & $10,6(\mathrm{M})$ & & \\
2016 & $85(\mathrm{M})$ & $12,6(\mathrm{M})$ & & \\
\hline
\end{tabular}

\section{Conclusiones}

La aplicación de medidas relacionadas con la implantación de caudales ecológicos en ríos temporales como el río Gaià genera procesos de autorecuperación del río cuyos efectos positivos se manifiestan sobre los indicadores de estado ecológico y otros indicadores de la complejidad estructural y biológica como los hábitats o cambios en las poblaciones de determinadas especies de fauna acuática como los peces, relevantes para adaptar la gestión del caudal ecológico a los requerimientos biológicos de las especies y hábitats relevantes.

La recuperación del caudal en un tramo que había estado totalmente seco desde hace años ha demostrado que un acuerdo de la administración con la industria es posible sin compensaciones económicas, que los caudales ambientales se pueden liberar sin afectar a los usos actuales y que la gestión a partir de una consigna de cotas se ajusta a las aportaciones naturales que el embalse recibe cada año, reflejando la temporalidad de la cuenca.

Con la restauración temporal de los caudales ecológicos se está consiguiendo la recuperación del funcionamiento del río como ecosistema, pero además se está consiguiendo la restauración de las relaciones afectivas de las poblaciones ribereñas con el río y su paisaje fluvial.

La coordinación y sinergias entre los diferentes actores del territorio es básica para poder sumar tantas iniciativas en la cuenca que permitan disponer de información científica y técnica de este nivel.

Asimismo, el desarrollo del proyectos de restauración pasiva como el de la alameda de Santes Creus ha demostrado la recuperación de la complejidad estructural y la diversidad biológica, mediante la evaluación de parámetros que no corresponden a aquellos establecidos para determinar el estado ecológico según la Directiva Marco del Agua, pero que aportan información complementaria de cómo evoluciona el sistema fluvial y que para ríos temporales es de gran utilidad para comprender como evolucionan los procesos fluviales.

Los indicadores biológicos e hidromorfológicos del Programa de seguimiento y control de la Agència no están representados en una escala geográfica idónea para poder evaluar cambios en el río por actuaciones de restauración morfológica, aunque se consideran elementales para poder evaluar las presiones y los impactos a nivel de masa de agua y por ende, de cuenca, y de esta forma planificar las medidas necesarias para alcanzar los objetivos de buen estado ecológico, como se ha podido realizar para este segundo ciclo de planificación.

\section{Agradecimientos}

Nuestro agradecimiento a Nuria Rovira, técnica medio ambiente de la Diputació de Tarragona, a Paolo Vezza, investigador del Politecnico de Turin, a MNConsultores y a la Asociación La Sínia por su colaboración cediendo datos que se han utilizado para la redacción de esta comunicación.

\section{$7 \quad$ Referencias}

Agència Catalana de l'Aigua, 2015. Plan de gestión del Distrito de Cuencas Internas de Catalunya. 2016-2021.

Agència Catalana de l'Aigua, 2011. Programa de seguimiento y control del DCFC. 2012-2018.

Diputación de Tarragona, 2016. Seguimiento hidrogeomorfológico y 
biológico del espacio de interés natural de la alameda de Santes Creus (Tarragona).

González del Tánago, M., Garcia de Jalón, D., 2007. Guía metodológica para la elaboración de proyectos. MIMAM.

Larned, S., Datry, T., Arscott, D., Tockner, K., 2010. Emerging concepts in temporary river ecology. Freshwater biology 55: 717-738.

Munné, A., Prat, N., Rieradevall, M., Solà, C., Bonada, N., 2003. A simple field method for assessing the ecological quality of riparian habitat in rivers and streams: QBR index. Aquat Conserv Mar Freshwat Ecosyst 13:147-167. 